\title{
Nonlinear Covariance for Multi-band Image Data
}

\author{
Imants D. Svalbe and Carolyn J. Evans \\ Department of Physics, Monash University, Melbourne 3168, \\ Australia.imants . svalbe@sci.monash .edu.au
}

\begin{abstract}
Multi-band data is often (unavoidably) pre-processed by nonlinear mappings, or is comprised of measurements taken across noncommensurate bands. We treat such cases using rank order statistics, avoiding problems of dimensionality and nonlinearity. Our aim is to reduce multi-band data to two images; one showing inhomogeneous image regions common to all of the image bands and another image which reflects the differences. The measure used is a nonlinear analog of linear covariance, the 'co-diversity', which responds to the relative homogeneity of local image regions in terms of rank. Algorithms to determine the 'co-diversity' are presented and applied to the interpretation of edges in multispectral data and to the combination of information from different sources, for example, binary and greyscale data. The method is robust to contrast variations across the data, but relies on some prior morphologic smoothing to ensure the local rank order is not dominated by noise.
\end{abstract}

Keywords nonlinear statistics, multivariate techniques, mathematical morphology

\section{Introduction}

For multi-band data there is no agreed method to apply morphologic processing because there is not a unique method of ordering multi-band data. Commensurate multi-band image data from a linear vector space can be mapped to form a single greyscale image which is then amenable to morphologic or other forms of processing. This can be done using R-ordering [1] using some distance metric or by artificially imposing a total ordering on vector data [2]. For noncommensurate multivariate data, where the component bands are dimensionally distinct, these techniques are not meaningful. Multi-band image data that has either suffered strong non-linear mapping on one or more components, or where individual bands are measures of different kinds of variables, cannot be arbitrarily combined using linear statistics or by some form of vector ordering approximation. Here we will adopt the use of relatively simple nonlinear statistics to help condense multi-band image data to a strongly representative greyscale form. 


\section{Rank and Linear Variance Measures}

We have considered, in some detail, the use of measures of linear and nonlinear variance as a means of enhancing inhomogeneous regions in a single component image in [3]. It was shown that previous rank variance indicators, like that associated with Spearman's rank correlation $\rho$ [4] and the R-estimate of variance discussed by Draper [5], are not very responsive to ties in image data and so poorly enhance image structure. Linear variance is sensitive to the numeric distribution of local grey values and the presence of outliers. The measure of nonlinear variance, called diversity, was characterised in [3] and was shown to be a useful method of enhancing inhomogeneous regions in single-component images.

\subsection{Diversity: Nonlinear, Tie-Sensitive Variance}

A measure of rank variance for $N$ (odd) data points can be defined by first ranking the data using the same method as Chung and Marden [6]. Rank 1 is assigned to the lowest value, rank 2 is assigned to the next lowest values and so on. The same integer ranks are assigned to tied values. The diversity of these ranks is then defined by

$$
d=\frac{\sum_{i=1}^{N}\left(r_{i}-r_{m e d}\right)^{2}}{N\left(N^{2}-1\right) / 12}
$$

where $r_{i}$ is the rank of the $i$ th value, $r_{m e d}$ is the assigned rank of the median value, and $N\left(N^{2}-1\right) / 12$ is a normalising factor.

The diversity of single-band image data is calculated using a moving, local window containing $N$ pixels. The ranking process must be repeated every time new data enters the moving window before the diversity can be calculated.

The diversity measure is invariant to any strictly order preserving mapping, for example, nonlinear contrast stretching. The diversity can be measured with respect to ranks other than the median rank; equally useful are measures of the rank distribution based on the local maximum rank, $r_{\text {max }}$, or local minimum rank, $r_{m i n}$. Diversity intrinsically responds tot he number of ties present in the local data values; the occurrence of more ties present implies greater homogeneity. For those cases where the selected local window size $N$, is greater than $g$, the number of possible grey values in an image, the maximum diversity of 1 cannot be attained as there will always be at least $N-g$ ties.

Because the chance of obtaining tied values depends in part on the precision of grey value specification (eg the number of bits per pixel), some form of local smoothing should be considered, or otherwise all local regions contain few or no ties and hence must be considered as being equally and maximally rough. Smoothing with an morphological open or close forces the replication of local maximum or minimum values (but introduces no new values). Such smoothing prevents the result being dominated by image noise. 


\section{Extension of Diversity to Multi-component Images}

We extend the idea of the diversity to a nonlinear measure called 'co-diversity', which can be applied to multi-component images. The co-diversity is made up of two terms; a cross diversity term $x d$ and a residue term, $\Delta$. Local $x d$ measurements made in a multi-band image highlight inhomogeneous image regions which are common to the input bands and local $\Delta$ measurements reflect the differences between the input bands. In this section two different methods of calculating co-diversity are presented.

\subsection{In-band Cross-diversity}

For multivariate data, the cross-band diversity, $x d$ can be measured by correlating the within-band $d$ values, obtained at some scale $N$, for each spatially registered point. A useful measure of $x d$ is the mean or median of the in-band $\mathrm{d}$ values at each point. Since the in-band diversity characterises the spatial in-band geometry at each point in each band, we can combine locally measured $d$ values using linear or non-linear statistics as necessary.

A residue measure for multi-band data $(\Delta)$ can be calculated by summing the squared deviations of the ranked values with respect to the mean or median rank value. This cross-band measure can be larger than $N\left(N^{2}-1\right) / 12$ by a factor of $(b-1) / 2$ for $\mathrm{b}$ bands, and so it, too, can be meaningfully normalised.

$$
\begin{aligned}
x d & =\operatorname{median}\left\{d_{i}\right\}, \quad i=1, . ., b . \\
\Delta & =\frac{\sum_{i=1}^{b}\left(d_{i}-x d\right)^{2}}{((b-1) / 2) N\left(N^{2}-1\right) / 12},
\end{aligned}
$$

This method is simple to implement and relatively fast to compute. The results are, however, sensitive to the global number of grey scales in each band $g$ if $g<N$, because diversity is used. Comparing in-band values this way ignores spatial mismatches over lengths smaller than the local window size. The crossband, in-place diversity measure takes these spatial mismatches into account.

\subsection{Cross-Band In-Place Diversity}

Now the rank values are replaced in their original local spatial order for each band and a pair-wise cross-product of the ranks is summed over the local window.

Here the ranks must not only be similar, but have the same spatial structure to contribute to a high value of $x d$. The residue $\Delta$ is calculated as the sum of pair-wise differences of the absolute value of the ranks. The general formula for cross ${ }^{2}$ band, in-place $x d$ and residue $\Delta$ are,

$$
\begin{aligned}
x d & =\frac{\sum_{j=1}^{b} \sum_{k=j+1}^{b} \sum_{i=1}^{N} a_{j k}\left(\left|r_{i j}-\left(r_{m e d}\right)_{j}\right| \cdot\left|r_{i k}-\left(r_{m e d}\right)_{k}\right|\right)}{(b-1)(b / 2) N\left(N^{2}-1\right) / 12} \\
\Delta & =\frac{\sum_{j=1}^{b} \sum_{k=j+1}^{b} \sum_{i=1}^{N} b_{j k}\left(\left|r_{i j}-\left(r_{m e d}\right)_{j}\right|-\left|r_{i k}-\left(r_{m e d}\right)_{k}\right|\right)^{2}}{(b-1)(b / 2) N\left(N^{2}-1\right) / 12},
\end{aligned}
$$


where $r_{i j}$ is the assigned rank at position $i$ in band $j$ and $\left(r_{m e d}\right)_{j}$ is the assigned rank of the median value in band $j .(b-1)(b / 2) N\left(N^{2}-1\right) / 12$ is a normalisation factor.

The co-diversity of multi-band image data is calculated using a moving, local window containing $N$ pixels. The ranking process must be repeated in each band every time new data enters the moving window before the co-diversity can be calculated.

The insertion of coefficients $a_{j k}$ enables compensation of each pair-wise contribution to give equal weight to $x d$ for the general case of bands $j$ and $k$ being any type of image. For example, if both images have $N$ grey scales, then $a_{j k}=1.0$. If one image was binary and the other $N$ levels, the maximum summed cross product is $\left(N^{2}-1\right) / 8$, so then $a_{j k}=2 N / 3$. If both images are binary, the maximum summed cross product is $(\mathrm{N}-1) / 2$, so $a_{j k}=N(N+1) / 6$.

The coefficients $b_{i j}$ similarly enable each pair-wise difference to give equal weight to $\Delta$. Similar weighting schemes can be applied for the in-band measures of section 3.1 to compensate for the effect of bands with reduced grey scales.

\section{Applications}

We applied the $x d$ measures to 3 band, RGB data to produce an 'intensity' image and cross-band variation, from which reliable 'colour' edges were able to be extracted using 'top-hat' morphologic ridge detectors. Where the residue $\Delta$ is strongly correlated to $x d$, the extracted edges in $\Delta$ and $x d$ may be combined using a point-wise maximum; for anti-correlated residues (where noise dominates the residue), the minimum of $x d$ and the residue can be used to suppress nonedge structures. When one band of inconsistent data is used in cross variance with 'correct' data, the residue will largely contain an image of the inconsistent band data. Figures $1 \mathrm{a}$ and $1 \mathrm{~b}$ show $x d$ and $\Delta$ for the in-band method, respectively; Figures $2 \mathrm{a}$ and $2 \mathrm{~b}$ show the results obtained using the in-place co-diversity measure.

Figures 1 and 2 can be compared to figure 3, which shows the local linear variance of the first and the last (third) principal components of the $R G B$ image respectively. The last principal component does not exactly show the disagreement between image bands, which $\Delta$ measures. Edges are highlighted in figure $3 \mathrm{~b}$ which also appear in figure $3 \mathrm{a}$, and which are present in more than one band of the original RGB image. On the other hand, the $x d$ and $\Delta$ images show complementary sets of information.

When calculating the co-diversity of a multi-band image, we have also extended the original 3-band data to include the open and close of each colour band, and then calculated $x d$ for these 9 bands. This results in a cleaner edge image, as the original data matches the open or close near edge locations, but is suppressed in flat regions, where the open and close, being smoothed, have low within-band $d$ values.

An orientation sensitive $x d$ and $\Delta$ values can be can be obtained by calculating $x d$ using 1-D oriented windows, eg. horizontal, vertical, and left and right 
diagonal, and taking the median or mean of the results as if they were generated from separate image bands.

By calculating (in-band) $x d$ values between a greyscale image $I$, and each member of a set of thresholded images $T(I)$, a global threshold $T$ can be selected which maximised the global mean value of $x d$. The thresholded image is selected on the basis of retaining the maximum degree of correlated spatial information with respect to the original grey level image.

\section{Conclusions}

The rank diversity, $d$, represents the wide range of possible variations of local geometries in a concise and descriptive fashion. Because this statistic is directly comparable for all images (even after strongly non-linear mappings or for separate bands of dimensionally independent variables), measurement of the common cross-band diversity, $x d$, makes it a convenient and robust method to combine any bands of spatially registered data. The residue, $\Delta$, provides a reliable measure of the between-band variation not registered in $x d$. These methods should prove of general use for the combination of spatial data such as economic, census and geographic information, as well as for multi-band image data (such as colour or satellite data) and for blending visual and range image data.

\section{Acknowledgements}

CJE would like to acknowledge the finanrial support of the CSIRO Australia's Division of Mathematical and Information Sciences and the Australian Research Council (through the APA award) in her PhD studies.

\section{References}

1. Barnett V.. The Ordering of Multivariate Data. J. R. Statist. Soc. A., 139 part 3. (1976), 318-344.

2. Jones R. and Talbot H.. Morphological Filtering for Colour Images with No New Colours. IVCNZ '96, 149-154, Lower Hutt, New Zealand, 1996.

3. Evans C.J. and Svalbe I.D.. Nonlinear Variance Measures in Image Data. To be presented at SPR 98.

4. Kendall M.G.. Rank Correlation Methods, 4th ed.. Griffin, London, 1970.

5. Draper D.. Rank-Based Robust Analysis of Linear Models.I. Exposition and Review. Statistical Science, 3(2) (1988), 239-271.

6. Chung L. and Marden J.I.. Use of Nonnull Models for Rank Statistics in Bivariate, Two-Sample, and Analysis of Variance Problems. J. American Statistical Association, 86(413) (1991), 188-200.

7. Serra, J.. Image Analysis and Mathematical Morphology. volume 2: Theoretical Advances. Academic Press, London, 1988. 


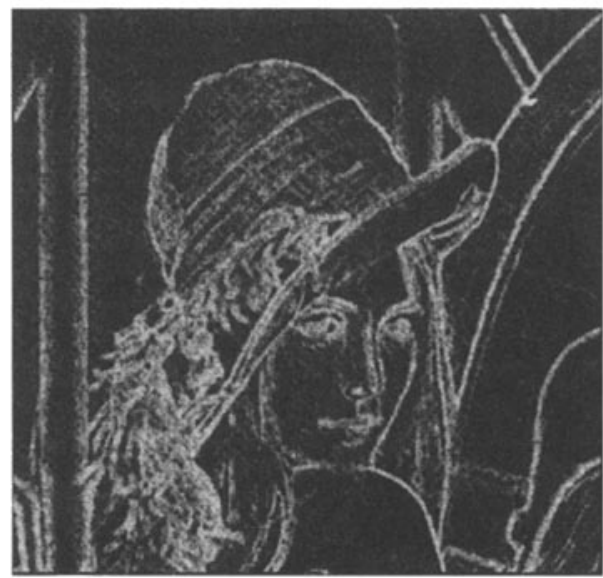

(a)

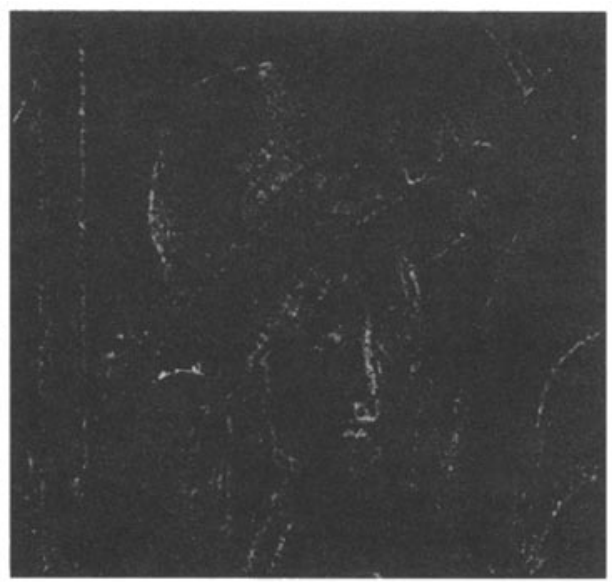

(b)

Fig. 1. (a) In-band $x d$ of RGB image of Lena, (b) In-band $\Delta$ of RGB image of Lena, both locally calculated in circular regions of diameter 7 pixels.

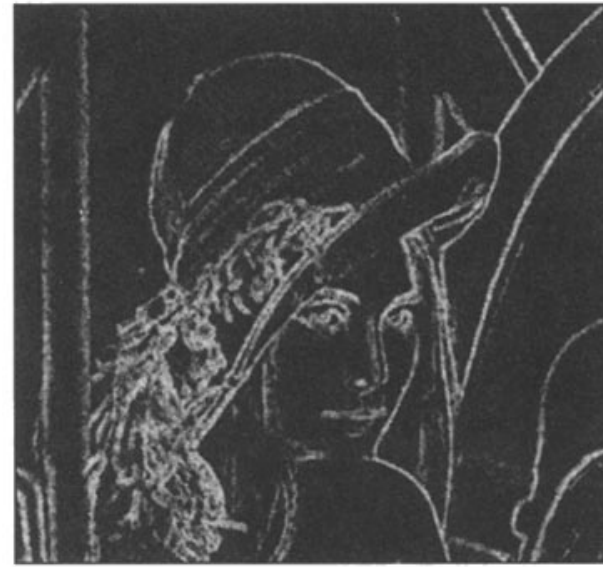

(a)

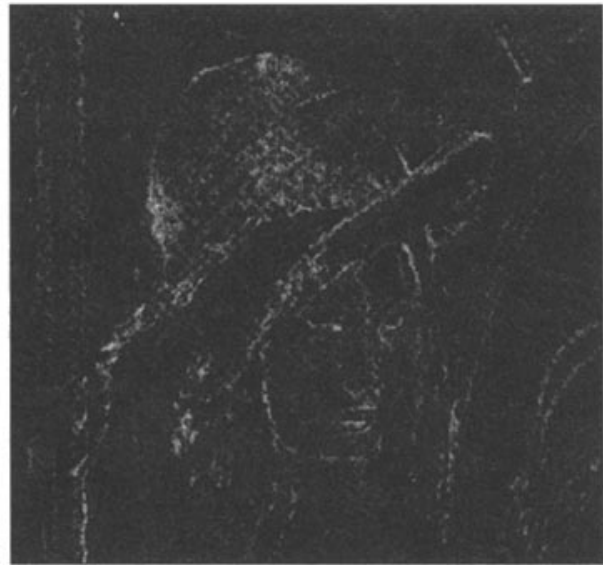

(b)

Fig. 2. (a) In-place $x d$ of RGB image of Lena, (b) In-place $\Delta$ of same image, both locally calculated in circular regions of diameter 7 pixels. 


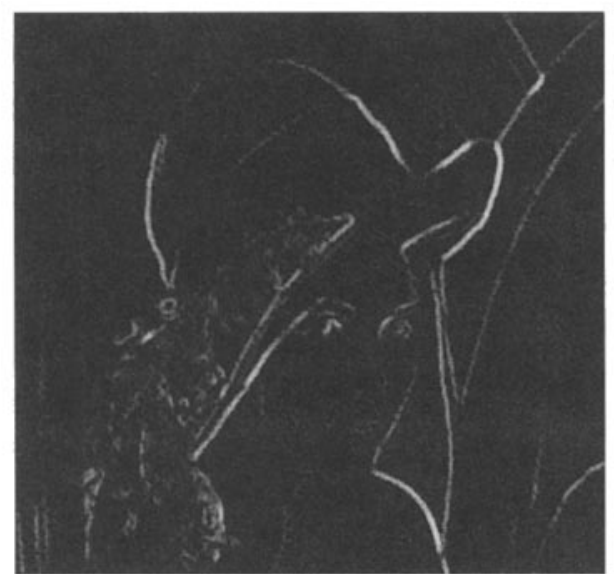

(a)

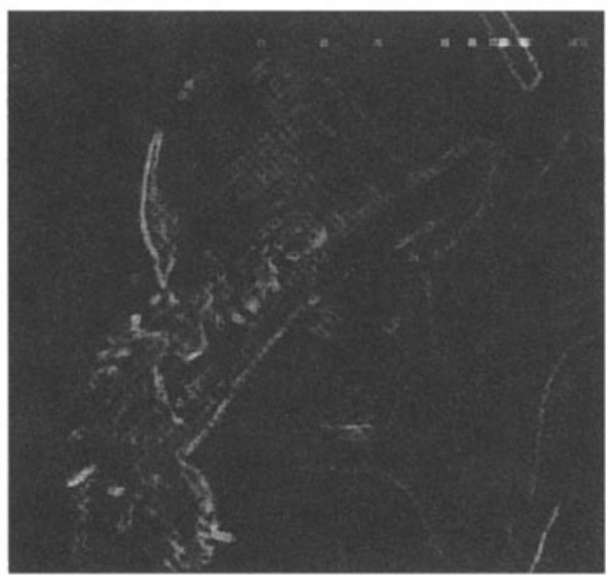

(b)

Fig. 3. Local linear variance of (a) the first principal component and (b) the last principal component of the RGB image of Lena. Local linear variance was calculated using a $7 \times 7$ window. 